\title{
Enhanced Dielectric Properties of Epoxy-based Photoresist Nanocomposites using Carbon-Coated Nickel Nanoparticles for High Voltage Integrated Capacitors
}

\author{
M. S. Alfonso ${ }^{1 *}$, C. Lapeyronie ${ }^{1}$, M. Goubet ${ }^{1,2}$, B. Viala ${ }^{2}$, J.-H. Tortai ${ }^{1^{*}}$ \\ ${ }^{1}$ University Grenoble Alpes, CNRS, CEA/LETI-Minatec, Grenoble INP, Institute of Engineering and \\ Management University Grenoble Alpes, LTM, Grenoble F-38054, France \\ ${ }^{2}$ University Grenoble Alpes, CEA, LETI, 38000 Grenoble, France \\ *Corresponding authors: (alfonsomarcosalvatore@gmail.com; jean-herve.tortai@ltmlab.fr)
}

\begin{abstract}
High- $k$ nanocomposite photoresists are highly sought-after dielectric materials for the manufacturing and miniaturization of integrated capacitors. Herein, in-depth high voltage dielectric characterizations of Metal Polymer Composites (MPCs) having carbon-coated nickel nanoparticles (Ni@C) dispersed into epoxy-based photoresist (SU-8) are reported.
\end{abstract}

The MPCs were fabricated using three-step formulation (deagglomeration, surface functionalization and dispersion in a polymeric matrix) involving simple sonochemical methods. Finally, films of MPCs were deposited on silicon wafers by spin-coating, and Metal-InsulatorSemiconductor (MIS) capacitors were fabricated. Formulations with increasing nanoparticles/polymer volume fractions were prepared in order to determine a previously unestablished percolation threshold with (Ni@C) nanoparticles. The experimental results were modeled using the generalized power-law equation of the percolation theory (PT) and were compared with effective medium approximation (EMA).

An enhancement of the complex dielectric permittivity of $\sim 116 \%$ was detected keeping a reasonable value of losses of 0.32 at $5 \mathrm{kHz}$. However, no giant permittivity in vicinity of the percolation threshold was detected. The high-voltage dielectric properties showed the occurrence of two electron field emission mechanisms, but no electrical aging or dielectric breakdown below 
$\pm 0.38 \mathrm{MV} / \mathrm{cm}$ occurred. This study shows a reliable wafer-scale film fabrication process of MPCs for the manufacturing of long-awaited miniaturization of high-voltage capacitors.

Keywords: Nano Composite, Nano particles, Core-shell nanostructures, Dielectric properties, Polymer-matrix composites

\section{Introduction}

New high-k dielectric nanocomposite photoresists for the implementation of miniaturized capacitors are highly sought-after materials by the microelectronics community (1-7). Achieving homogeneous Metal Polymer Composites (MPCs) with metal nanofillers would represent a real breakthrough for microelectronics applications. Despite this, obtaining homogeneous high-k MPCs still remains a pivotal challenge in the field. Previous reports demonstrated that MPCs can exhibit large-to-giant permittivity in the vicinity of the percolation threshold $(8,9)$.

However, percolative nanodielectrics usually suffer by large increases of conductivity causing large dissipation factors, incompatible with their practical applications. The main issue is represented by the fact that metal nanoparticles are hardly ever uniformly dispersed in the polymeric host. Metal nanoparticles usually form conductive aggregates with large ohmic loss, which dramatically rises the dissipation factor. Hence, the most relevant challenge consists in combining high permittivity and acceptable loss tangent for a capacitor.

Few examples of MPCs have shown so far that high permittivity and low dissipation factors are achievable using Nickel nanoparticles. Nevertheless, the published values vary in a wide range according to the size, shape and volume fraction of the nanocomposite employed.

For example, Dang et al. (10) reported that Ni/PVDF nanocomposites can be used as a high-k material for energy storage applications with a dielectric constant of 400 at $100 \mathrm{~Hz}$ and losses of 0.18 at a nanoparticle/polymer volume fraction of 17 vol\%. Even greater values were reported by Zhang et al. (11) for Ni/P(VDF-TrFE) nanocomposites with a record value of about 1000 at $1 \mathrm{kHz}$ with losses of 0.9 for a nanoparticle/polymer volume fraction of 53 vol\%. In contrast, other studies 
showed moderate values of permittivity of about 200 for $\mathrm{Ni} / \mathrm{PU}$ nanocomposites, nevertheless reaching significantly lower losses $(0.005$ at $1 \mathrm{kHz})$ for a nanoparticle/polymer volume fraction of 25 vol\% (12).

In this view, Carbon-coated Nickel nanoparticles ( $\mathrm{Ni@C)}$ are a promising system. Firstly, the metallic core (i.e. conductive inclusions) is efficiently protected against oxidation by a carbon shell having a graphene-like structure. Secondly, the graphene-like shell offers the possibility to easily functionalize the nanoparticles surface. This may be beneficial for improving the nanoparticles dispersion in the polymeric matrix. Achieving a homogeneous dispersion of $\mathrm{Ni}$ nanoparticles in a polymeric matrix is not straightforward mainly because of their magnetism. Therefore, controlling the homogeneity of the dispersion still represents a significant challenge (13-16). In order to achieve this, we present a detailed study of sonodispersion protocols of nanoparticles in solution aiming to minimize particle aggregation.

The aim of this work is to establish a wafer-scale film fabrication process for Metal-InsulatorSemiconductors (MIS) using metal polymer nanocomposites (MPC) based on commercial carboncoated nickel nanoparticles (Ni@C) embedded in epoxy-based photoresist SU-8 for the miniaturization of high-voltage capacitors. The capacitive behavior of the formulated nanocomposite films have been investigated by using broadband dielectric spectroscopy and high voltage dielectric measurements. High voltage C-V loops showed a processable voltage window, narrowed down when the nanoparticles loading increases, but no evidences of electrical aging or irreversible dielectric breakdown was encountered. High-voltage dielectric properties were also reported revealing two electron field emission mechanisms. The dielectric responses of the formulated nanocomposites were modelled by using the percolation theory (PT) and the effective medium approximation theory (EMA). Nevertheless, only the percolation theory well matched with the experimental data, offering indication on the percolation threshold (close to 1 vol\%). 
The resulting percolation threshold is low and it is mainly due to the chain-like arrangements of carbon-coated Nickel nanoparticles (Ni@C), which occurs during the film fabrication process of Metal-Insulator-Semiconductor (MIS) as validated by cross section SEM images of the films.

We concluded that the perpendicular chain-like agglomerates of Ni@C in the MPC films might be held responsible of the low percolation threshold, which strongly penalized the improvement of dielectric properties. However, this work provides a new path to improve the dielectric properties of nanocomposite photoresists and a wafer-scale film fabrication suitable for long-awaited miniaturization of high voltage capacitors.

\section{Experimental Part}

\subsection{Materials}

Commercial (Ni@C) nanopowders with a nanoparticle mean diameter of $20 \mathrm{~nm}$ were purchased from Nanostructured \& Amorphous Materials, Inc.. Poly(styrene), $\Omega$-(1-Pyrenyl)-Terminated $\left(M_{W} / M_{N} 1.3\right)$ (PyrPS) supplied by Polymer Source was used as a surface agent to functionalize the (Ni@C) nanoparticles. Negative epoxy photoresist SU-8 (2100) was supplied by MicroChem Corp. The photoresist mixture is composed of epoxy resin, propylene carbonate, triaryl sulfonium initiator and cyclopentanone as solvent in different ratio depending on its formulation. Propylene glycol methyl ether acetate $>99 \%$ (PGMEA) was used as solvent and purchased from Microposit ${ }^{\mathrm{TM}}$ EC Solvent.

\subsection{Nanocomposite preparation}

The objective of this preparation step is to produce a homogeneous dispersion of nanoparticles in the photoresist in order to deposit thin films on silicon wafers by spin-coating. Direct mixing of the nanopowders in polymeric host solution usually leads to inhomogeneous dispersions and dewetting during spin-coating (17). In order to overcome this issue, we developed a multistep 
procedure involving ultrasonication methods followed by surface functionalization of the nanoparticles prior to film deposition. We aimed to obtain a non-covalent surface functionalization of the carbon-coated metallic nanoparticles in order to avoid damaging the protective graphene-like shell (18). The functionalization was performed with Pyrene-terminated polystyrene (PyrPS) under ultrasonication, in order to obtain a thin nanometric coating of an electrical insulator on the (Ni@C) metallic nanoparticles. This step was also used in order to provide a better dispersibility of the nanoparticles in the polymeric host matrix. In addition, this polymeric coating allows to avoid additional direct exposure of the carbon shell to sonication damage during the last step of dispersion of the nanoparticles into the negative epoxy photoresist (13). Our three-step protocol is illustrated in Figure 1.

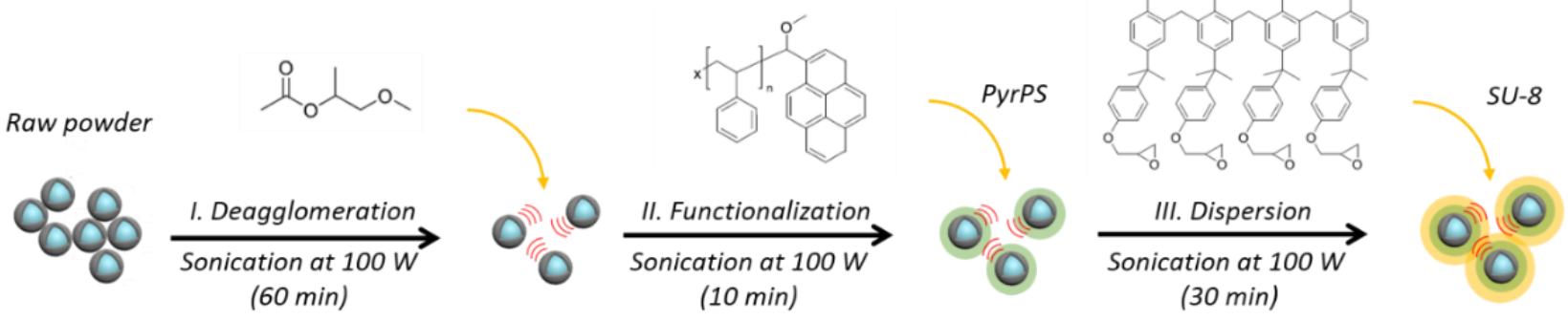

Figure 1 - Three-step sonodispersion protocol. Deagglomeration (step 1), surface functionalization (step 2), and dispersion in the polymeric matrix (step 3).

Step 1- Nanopowder deagglomeration: $1 \mathrm{~g}$ of powder (as-received) was poured in $10 \mathrm{ml}$ of PGMEA and sonicated for 60 minutes at $100 \mathrm{~W}$ by using a VCX500 ultrasonic processor (Sonics). Step 2- Surface functionalization: $100 \mathrm{mg}$ of PyrPS was added to the colloidal suspension obtained in Step 1 and for sonicated 10 further minutes at the same power. Step 3- Dispersion into photoresist: according to the desired volume fraction of nanoparticles in the film (after solvent evaporation), known volumes of functionalized colloidal suspension and SU-8 photoresist were 
mixed and sonicated for 30 more minutes at the same power. The formulated nanocomposite volume fractions were determined using the following equation:

$$
\% v=\frac{\frac{C_{N p} * V_{N p}}{d_{N p}}}{\frac{C_{N p} * V_{N p}}{d_{N p}}+\frac{V_{R e s i n} * d_{R e s i n} * \%_{d r y}}{d_{d r y}}}
$$

where: $C_{N p}$ is the initial concentration of $\mathrm{Ni@C} \mathrm{dispersion,} V_{N p}$ its volume and $d_{N p}$ is the true bulk density value of Ni@C nanopowders equal to $8.908 \mathrm{~g} / \mathrm{ml}$, while $V_{\text {Resin }}$ is the initial volume of SU-8 resin, $d_{\text {Resin }}$ is its density whereas $d_{d r y}$ and $\%_{d r y}$ represent the density of dry SU-8 resin and its dry percent fraction, respectively. Note that the amount of PyrPS was considered negligible. The nanocomposite solution was spin-casted within 30 minutes of preparation in order to avoid any sedimentation of the functionalized nanoparticles.

\subsection{Film deposition}

Nanocomposite films were prepared by spin-coating on 4-inches Boron Doped p-type silicon wafers (purchased from BT Electronics) with a resistivity of 0.001-0.003 Ohm.cm. The targeted thickness of the films is in the range of 5-15 $\mu \mathrm{m} .4 \mathrm{~mL}$ of nanocomposite solution were poured in the center of the wafer before spinning at $1000 \mathrm{rpm}$ with an acceleration of $300 \mathrm{rpm} / \mathrm{s}$ for 30 seconds. Subsequently, the films were baked at $95^{\circ} \mathrm{C}$ for $3 \mathrm{~min}$ in order to remove the solvent.

The films were cured by 20 s UV exposure using a MJB4 Mask Aligner (Power density: 300 $\mathrm{mJ} / \mathrm{cm}^{2}$ ). A home-built mask protected two areas of the deposited nanocomposite film, in order to obtain two large edges at the center of wafer to measure the film thickness (Figure 2). The final post-exposure bake was performed at $95{ }^{\circ} \mathrm{C}$ for $3 \mathrm{~min}$. The last baking step was used to crosslink the negative epoxy-base photoresist SU-8 in correspondence of the UV-exposed areas. Finally, the films were developed by immersion of the wafer in PGMEA in order to remove the non-exposed film areas (without electrodes). Finally, $30 \mathrm{~nm}$-thick circular gold top electrodes were deposited by 
Physical Vapor Deposition (PVD) at a deposition rate of $0.5 \mathrm{~nm} / \mathrm{sec}$ through a shadow mask. Three different electrode diameters $(2.5,1.5$ and $0.75 \mathrm{~mm})$ were used in order to increase the accuracy of the permittivity measurements.

Film thickness was measured with a stylus profilometer (Bruker), by repeating the measurement six times along both edges of the unexposed area of the films. The thickness showed to depend on the ratio between the volume of the nanoparticles suspension and the volume of photoresist for a known volume fraction in the film, as the viscosity showed to depend on the composition. The average film thickness and electrode surface values are summarized in Table 1 . These values were used for calculating the capacitance of the devices using the plate capacitor formula. The metal insulator semiconductor (MIS) capacitors fabrication with MPCs dielectric photoresist is illustrated in Figure 2.

(a)

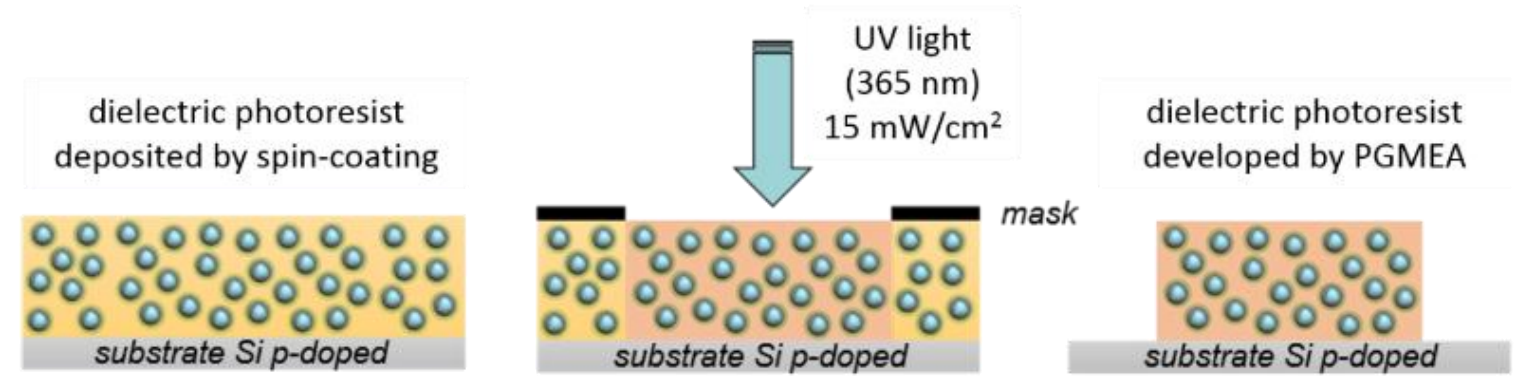

(b)

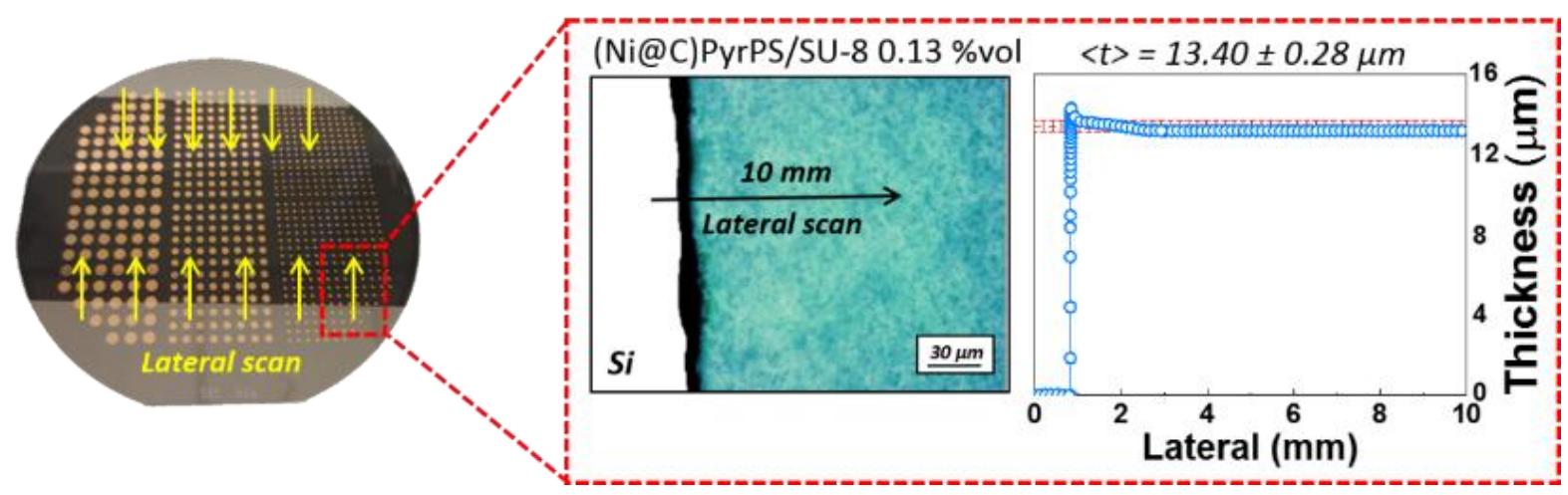

Figure 2 - Metal-Insulator-Semiconductor (MIS) Implementation. (a) Schematic of the fabrication procedure of the UV-cured dielectric nanocomposite thin films. (b) Control of the top surface quality by optical microscopy and thickness measurement by stylus profilometer. 
Table 1 - Thickness values of each dielectric nanocomposite film (thickness error is calculated as standard deviation of six scan measurements on both edges of unexposed film).

\begin{tabular}{ccccc}
\hline & Average Thickness $<\mathrm{t}>(\mu \mathrm{m})$ & \multicolumn{3}{c}{ Surface electrode $\mathrm{A}\left(\mathrm{mm}^{2}\right)$} \\
\hline Pure Epoxy-based SU-8 & $10.18 \pm 0.27$ & $4.9 \pm 0.029$ & $1.8 \pm 0.029$ & $0.4 \pm 0.008$ \\
(Ni@C)PyrPS/SU-8 (0.13 vol\%) & $13.40 \pm 0.28$ & $4.9 \pm 0.033$ & $1.8 \pm 0.027$ & $0.4 \pm 0.003$ \\
(Ni@C)PyrPS/SU-8 (0.23vol\%) & $15.54 \pm 0.13$ & $4.9 \pm 0.049$ & $1.8 \pm 0.039$ & $0.4 \pm 0.013$ \\
(Ni@C)PyrPS/SU-8 (0.51vol\%) & $10.38 \pm 0.30$ & $4.9 \pm 0.033$ & $1.8 \pm 0.029$ & $0.4 \pm 0.009$ \\
(Ni@C)PyrPS/SU-8 (0.61 vol\%) & $8.26 \pm 0.34$ & $4.9 \pm 0.043$ & $1.8 \pm 0.027$ & $0.4 \pm 0.012$ \\
(Ni@C)PyrPS/SU-8 (0.87 vol\%) & $6.81 \pm 0.49$ & $4.9 \pm 0.029$ & $1.8 \pm 0.030$ & $0.4 \pm 0.014$ \\
(Ni@C)PyrPS/SU-8 (1.34 vol\%) & $4.8 \pm 0.21$ & $4.9 \pm 0.031$ & $1.8 \pm 0.028$ & $0.4 \pm 0.008$ \\
\hline
\end{tabular}

\subsection{Characterization}

Nanoparticle size and morphology were characterized by means of photon correlation spectroscopy (Zetasizer Ultra, Malvern Analytical) and transmission electron microscopy (TEM - FEI Tecnai Osiris S/TEM) operating at 200kV. The microstructure and morphology of nanocomposite thin films were characterized by scanning electron microscopy (SEM - Zeiss Ultra-Plus) using crosssections over several points on a wafer (from center to mid-radius).

The low-voltage dielectric properties of the dielectric nanocomposite films were determined by impedance measurements (Novocontrol Alpha-A Analyzer). The AC voltage was set at $\pm 3 \mathrm{~V}$ and the frequency range between $10^{2} \mathrm{~Hz}$ and $10^{6} \mathrm{~Hz}$. Raw impedances were post processed in order to subtract parasitic impedance contributions coming from cables and probes. The high-voltage dielectric properties of the films were measured at a fixed frequency $(5 \mathrm{kHz})$ with an AC voltage $( \pm$ $20 \mathrm{~V}$ ) and a superimposed DC bias voltage up to $\pm 380 \mathrm{~V}$ using a TF analyzer 2000E (aixACCT). Leakage current density was measured using a Keithley model 2400 source meter.

\section{Results and Discussion}

\subsection{Analysis of the dispersion quality of the colloidal suspension}


All preparation steps of the nanocomposite photoresists were characterized by estimating the quality of the dispersion of the nanoparticles in the liquid by optical transmission microscopy (Olympus BX53-P Polarizing Microscope).

A drop of the colloidal suspensions was deposited between two glass slides. Figure 3 shows how successive steps of sonodispersion allow the deagglomeration of the (Ni@C) nanopowders.

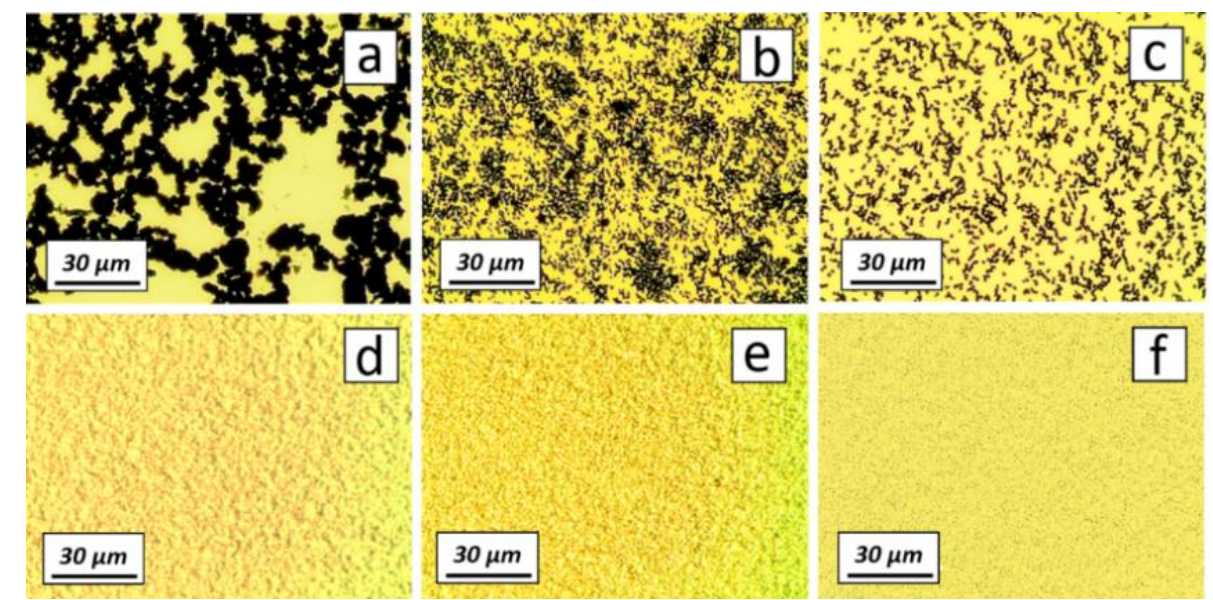

Figure 3 - Optical transmission microscopy of (Ni@C) colloidal suspension. (a) (Ni@C) 0.1 g/ml in PGMEA before sonication, (b) after 30 min of sonication, (c) after 40 min of sonication, (d) after 60 min of sonication, (e) (Ni@C) $0.1 \mathrm{~g} / \mathrm{ml}+$ PyrPS $0.01 \mathrm{~g} / \mathrm{ml}$ in PGMEA after 70 min of sonication, (f) (Ni@C) $0.05 \mathrm{~g} / \mathrm{ml}+$ PyrPS $0.005 \mathrm{~g} / \mathrm{ml}$ in SU-8 after 120 min of sonication by addition of SU-8 in 1:1 ratio.

Step 1: At the beginning, it was possible to observe the aggregation state in large clusters with an average diameter of $5 \mu \mathrm{m}$ (Figure 3.a). These agglomerates dislocated and formed long chains after 30 minutes of ultra-sonication (Figure 3.b). With increasing ultra-sonication time, the long chains tended to break and dispersion was observed (Figure 3.c), but with still residual observable chain-like agglomerates. Finally, after 60 min of sonication, the dispersion of ( $\mathrm{Ni@C)} \mathrm{nanoparticles}$ in the liquid appeared to be homogeneous (Figure 3.d). Longer sonication time were not studied in order to avoid damaging the graphene-like shell of nanoparticles. The latter is key to prevent the oxidation of the metallic core of the nanoparticles (13). Step 2: The functionalizing polymer agent 
PyrPS was attached to the surface of ( $\mathrm{Ni@C)}$ with 10 more minutes of ultra-sonication improving the degree of nanoparticles dispersion avoiding their further aggregation (Figure 3.e).

Step 3: The final step consisted in the dispersion of a known volume of ( $\mathrm{Ni@C)PyrPS} \mathrm{nanoparticles}$ in a known volume of epoxy-based photoresist SU-8. For example, the 1:1 mixture ratio corresponded to a volume fraction of 2.0 vol\% of (Ni@C)PyrPS in SU-8 in the film (after solvent removal).

TEM images of (Ni@C) nanoparticles after sonication in step 1 are shown in Figure 4.a. The images revealed polydisperse nanoparticles with diameters ranging from 10 to $35 \mathrm{~nm}$. Furthermore, it was visible that even at low concentrations (below $0.1 \mathrm{mg} / \mathrm{ml}$ ) these nanoparticles tended to form chain-like agglomerates with dimensions of the order of $500 \mathrm{~nm}$ in length probably due to magnetostatic interactions. The carbon layers surrounding the nanoparticles were visible, with a thickness of a few layers (3-5 nm). Nevertheless, this protecting carbon shell was less visible on the smallest nanoparticles.

The DLS results (shown in Figure 4.b) indicated the hydrodynamic radius $(\mathrm{nm})$ of the colloidal suspension. The Intensity distribution (black curve) revealed a main peak a $108 \mathrm{~nm}$ that is not symmetric, which may be attributed to the residual chain-like ( $\mathrm{Ni@C)}$ agglomerates after 60 minutes of ultra-sonication. The Number distribution (blue curve) revealed a peak at $23 \mathrm{~nm}$ relative to the separated nanoparticles. Finally, the Volume distribution (red curve) exhibited multiple peaks indicating the extra contribution of each population of aggregates.

(a)

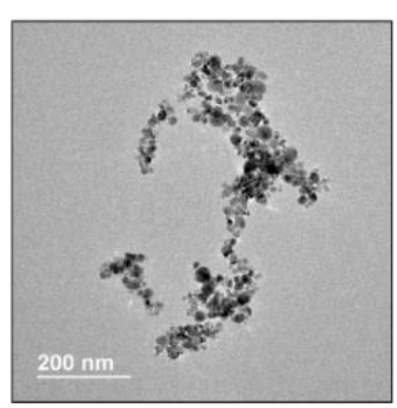

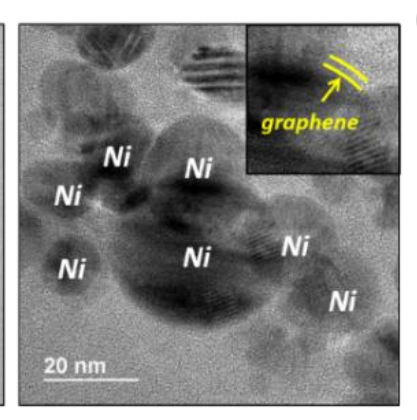

10 (b)

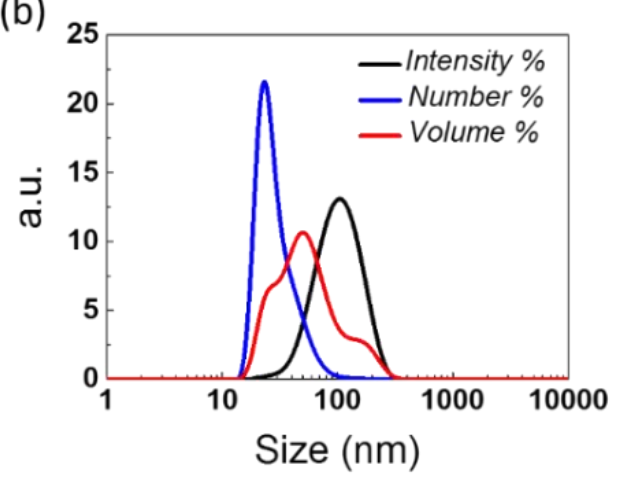


Figure 4 - Nanoparticles size investigation. (a) Transmission Electron Microscopy images (bright field, 200 kV) of (Ni@C) colloidal dispersion and high magnification of (Ni@C) aggregates (inset: graphene-like shell). (b) Size distribution curves obtained from DLS data: Intensity distribution (black curve), Number distribution (blue curve), Volume distribution (red curve).

\subsection{Morphology of the nanocomposite films deposited by spin-coating}

SEM cross-sectional films images of the (Ni@C)PyrPS/SU-8 films obtained at 0.87 vol\% nanoparticle/polymer ratio are shown in Figure 5.a. The film exhibited a low surface roughness and a local thickness of $7.2 \mu \mathrm{m}$ in agreement with profilometry measurements. Moreover, nanoparticles were present throughout the whole thickness of the film.
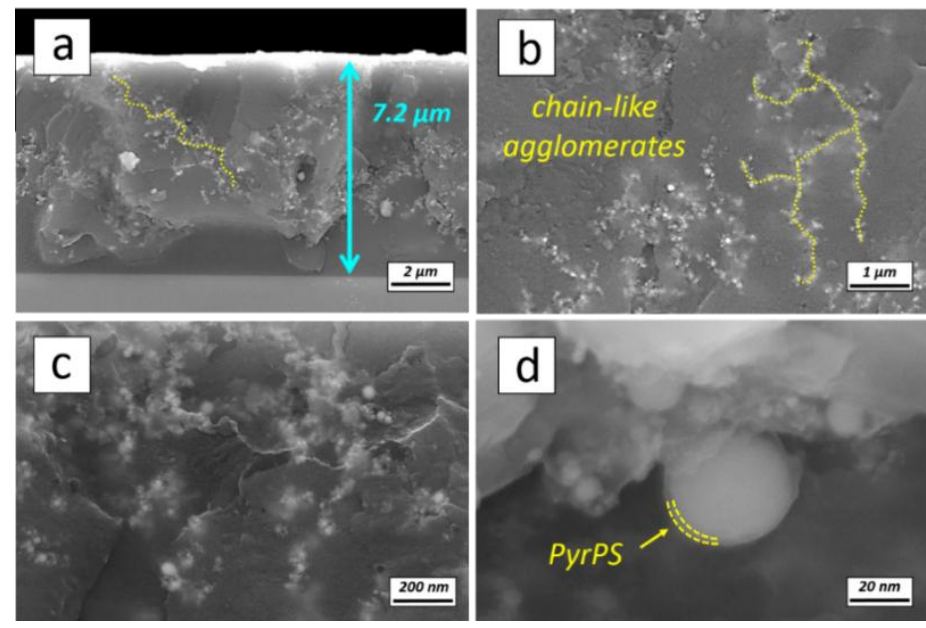

Figure 5 - Scanning electron microscopy images of percolative nanodielectric film. SEM images of the (Ni@C)PyrPS/SU-8 nanocomposite obtained at a 0.87 vol\% nanoparticle/polymer ratio. (a) Cross-sectional view of a $7.2 \mu \mathrm{m}$ thick film, (b) nanostructured chain-like agglomerates. (c) High magnification of nanostructured agglomerates (d) PyrPS functionalized nanoparticle.

No cracks, intended as longitudinal rifts along the entire thickness of the film or the lack of adhesion with the substrate were observed. In detail, high magnification images (Figure 5.b) revealed the presence of long chain-like agglomerates at 0.87 vol\% nanoparticle/polymer ratio (highlighted with yellow dots in Figure 5.b). Chain-like agglomerates were present in various size, ranging from $500 \mathrm{~nm}$ to few-microns. These arrangements are particularly unwelcome, as they 
may constitute a perpendicular percolative pathway network between the electrodes through the dielectric.

Figure 5.d shows the presence of a thin layer of PyrPS adsorbed on a (Ni@C) nanoparticle surface intended to increase particle electrical insulation. With this additional insulating layer surrounding the nanoparticles, ohmic conduction along the chain-like agglomerates might be reduced or cancelled. Further studies with involves higher $\mathrm{Mw} / \mathrm{Mn}$ functionalizing agent polymer ratio are underway in order to test higher filling fractions to obtain a closely spaced disordered (Ni@C)PyrPS/SU-8 nanocomposite.

\subsection{Dielectric spectroscopy}

The dielectric properties of the (Ni@C)PyrPS/SU-8 nanocomposite films were characterized between $100 \mathrm{~Hz}$ and $1 \mathrm{MHz}$ at room temperature without applying a DC bias. The influence of the (Ni@C) loading on the dielectric properties of nanocomposites is shown in Figure 6. The results have been averaged over 72 capacitors of different size. Sampling over large areas of the film allowed to verify the nanoparticles distribution. No significant variation of the dielectric properties was detected, thus confirming an equal distribution of the nanoparticles within the nanocomposites. Moreover, the dielectric properties were determined considering the uncertainty of the nanofabrication processes of capacitors, as follows.

$$
\frac{\Delta \varepsilon_{r}}{\overline{\varepsilon_{r}}}=\frac{\Delta C_{p}}{C_{p}}+\frac{\Delta t}{\bar{t}}+\frac{\Delta A}{A}
$$

Where $\frac{\Delta C_{p}}{C_{p}}$ is the limit of detection of impedancemeter, $\frac{\Delta t}{\bar{t}}$ represent the uncertainty on the films thickness and $\frac{\Delta A}{A}$ the uncertainty on the top surface electrodes.

As shown in (Figure 6.a), the permittivity of epoxy-based photoresist SU-8 increased with the addition of ( $\mathrm{Ni@C)} \mathrm{nanoparticles.} \mathrm{For} \mathrm{instance,} \mathrm{the} \mathrm{permittivity} \mathrm{at} 5 \mathrm{kHz}$ increased from 4.04 for pure epoxy-based photoresist SU-8 up to 8.72 at 0.87 vol\% nanoparticles/polymer ratio. Note that 
the dielectric properties of a formulated SU-8 film with addition of only PyrPS exhibited equal trends of the epoxy-based polymer matrix. This enhancement in the permittivity could be explained by interfacial polarization in accordance with the Maxwell-Wagner-Sillars theory (1925). In detail, the effect of an external electric field leads to an accumulation of electric charges at the interface between metallic ( $\mathrm{Ni@C)}$ nanoparticles and the dielectric matrix. As epoxy-based photoresist SU-8 is an excellent dielectric, the accumulated charges should not circulate and evacuate towards the electrodes, leading to an increase of the capacitance and thus of the permittivity of the nanocomposite.

(a)

(c)
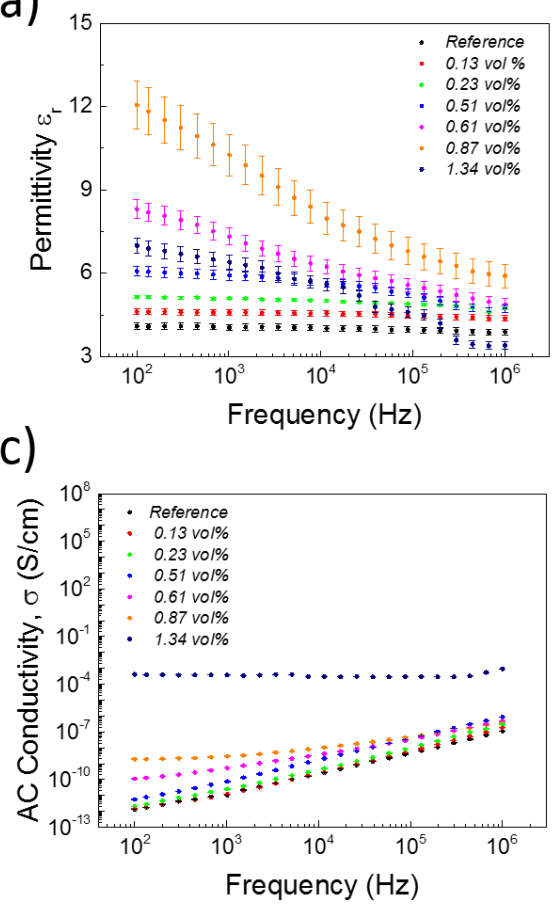

(b)

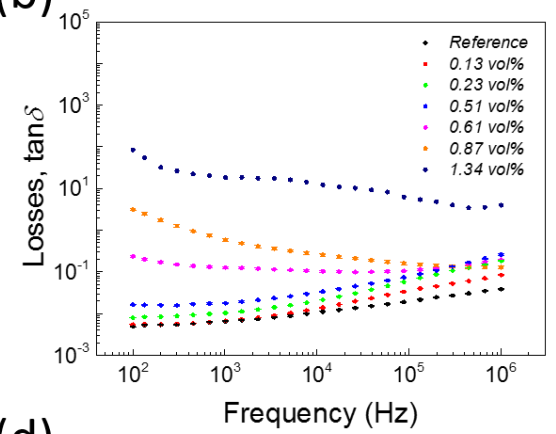

(d)

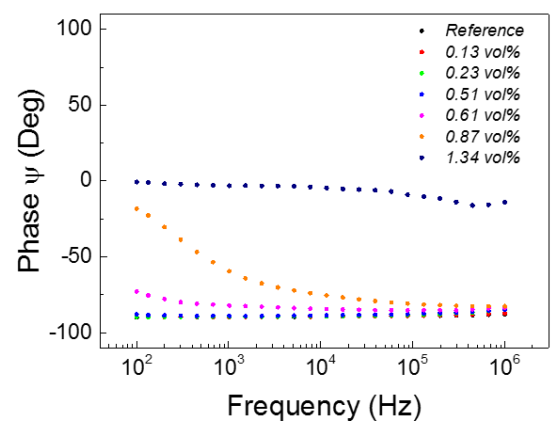

Figure 6 - Dielectric spectroscopy of (Ni@C)PyrPS/SU-8 nanocomposites. The frequency dependence of relative permittivity, (a), loss tangent, (b), AC conductivity, (c) phase angle (d) of (Ni@C)PyrPS/SU-8 nanocomposites with different filling fractions with a solicitation AC voltage of $\pm 3 \vee$ at room temperature.

This enhanced capacitive effect could be also understood through the image of a network of local nanocapacitors (26-31). The frequency dependence of dielectric losses expressed as $\tan \delta$ is 
shown in Figure (6.b). At $5 \mathrm{kHz}$, tan $\delta$ dramatically increased from $8.90 \times 10^{-3}$ for pure epoxy based photoresist SU-8 up to $3.22 \times 10^{-1}$ for the nanoparticle/polymer volume fraction of 0.87 vol\%. Above a volume fraction of 0.51 vol\%, $\tan \delta$ decreased as the frequency increased. This is unusual for a dielectric: more frequently, dielectric trends are observed below this value with an increase of $\tan \delta$ when the frequency increases. This change revealed a transition between dielectric and conductive regimes, which is mainly visible at low frequencies. The further increase of the volume fraction to 1.34 vol\% revealed a decrease in the capacitive behavior of the film exhibiting conductivity, losses and phase values clearly indicating the crossing of the percolation threshold of the system. This suggested that the percolation threshold of the system is low and between 0.87 vol\% and 1.34 vol\%. According to the SEM images, we can assume that the long perpendicular chain-like agglomerates of ( $\mathrm{Ni@C)} \mathrm{nanoparticles} \mathrm{could} \mathrm{provide} \mathrm{percolative} \mathrm{paths} \mathrm{inside} \mathrm{the} \mathrm{epoxy-}$ based photoresist SU-8 through which charges can circulate between top and bottom electrodes. The trend of the phase shift of the capacitors is shown in Figure (6.d). At high frequency, phase shifts were close to the theoretical value of $-90^{\circ}$ of an ideal capacitor including high volume fractions. At low frequency, the phase shift deviated from $-90^{\circ}$ above 0.51 vol\%. to reach a value close to zero at 1.2 vol\%. A phase shift of zero, is usually found with a conductor. This change was also in agreement with a transition between dielectric to conductive regimes above a volume fraction threshold comprised between 0.51 and 0.61 vol\%. The dielectric conductivity is shown in addition in Figure (5.c). Consistently with the phase shift, the dielectric conductivity increased from $1.02 \times 10^{-10} \mathrm{~S} / \mathrm{cm}$ for pure SU-8 up to $6.30 \times 10^{-9} \mathrm{~S} / \mathrm{cm}$ at 0.87 vol\%.

Finally, we report near-ideal capacitors below a threshold comprised between 0.51 and 0.61 vol\% with (Ni@C)PyrPS dispersed in SU-8 and leaky capacitors above the threshold. The leaky capacitors could be modelled with an ohmic resistor in parallel to the nanodielectric capacitor, the ohmic 
resistor being a representation of the conductive (Ni@C) chain-like agglomerates observed through the SU-8 matrix. The dielectric properties measured at $5 \mathrm{kHz}$ are summarized in Table 2.

The experimental data suggest that at high frequency, space charges were mainly stored at the particle/polymer interface and they did not have sufficient time to move. This means that the nanocomposite behaved as an almost perfect dielectric material. In contrast, at low frequency the resistive behavior became dominant.

Table 2 - The values of each dielectric nanocomposite film measured at $5 \mathrm{kHz}$.

\begin{tabular}{ccllc}
\hline @ $5 \mathrm{kHz}$ & $\varepsilon_{\mathrm{r}}$ & $\tan \delta$ & $\sigma(\mathrm{S} / \mathrm{cm})$ & $\Psi_{\text {Phase }}\left({ }^{\circ} \mathrm{Deg}\right)$ \\
\hline Pure Epoxy-based SU-8 & 4.04 & $8.90 \times 10^{-3}$ & $1.02 \times 10^{-10}$ & -89.62 \\
(Ni@C)PyrPS/SU-8 (0.13 vol\%) & 4.56 & $1.03 \times 10^{-2}$ & $1.04 \times 10^{-10}$ & -89.53 \\
(Ni@C)PyrPS/SU-8 (0.23 vol\%) & 5.04 & $1.59 \times 10^{-2}$ & $1.70 \times 10^{-10}$ & -89.38 \\
(Ni@C)PyrPS/SU-8 (0.51 vol\%) & 5.79 & $2.58 \times 10^{-2}$ & $6.80 \times 10^{-10}$ & -88.80 \\
(Ni@C)PyrPS/SU-8( 0.61 vol\%) & 6.52 & $1.11 \times 10^{-1}$ & $2.07 \times 10^{-9}$ & -83.82 \\
(Ni@C)PyrPS/SU-8 (0.87 vol\%)) & 8.72 & $3.22 \times 10^{-1}$ & $6.30 \times 10^{-9}$ & -72.15 \\
(Ni@C)PyrPS/SU-8 (1.34 vol\% & 5.81 & $1.62 \times 10^{1}$ & $4.12 \times 10^{-6}$ & -3.520 \\
\hline
\end{tabular}

In this case, the nanocomposite was comparable to a leaky dielectric polymer characterized by a dielectric permittivity and a dielectric conduction. When the conduction became too large, the capacitor could be short-circuited and became a high-value resistor. The circulation of charges from or towards the electrodes may be increased by the presence of long chain-like agglomerates of nanoparticles that are mainly perpendicular to the electrodes.

(a)

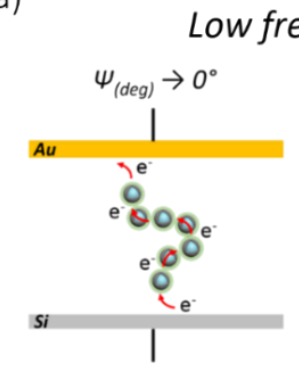

ow frequency

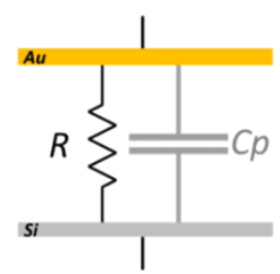

(b)

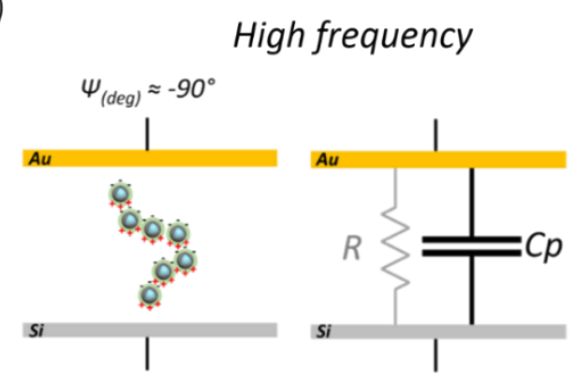

Figure 7 - Schematic electrical circuit equivalent - Synthetic representations of the (Ni@C)PyrPS/SU-8 nanocomposite at low frequency (a) and high frequency (b), respectively with perpendicular long chain-like agglomerates in vicinity of percolation threshold. 
This type of perpendicular percolation pathway could be responsible of additional charge injection or charge draining from or to the electrodes during the polarization of the films. Figure 7 shows two schematic equivalent electrical circuits of the nanocomposite at low and high frequencies, respectively. The permittivity and the conductivity dependence on the nanoparticles volume fraction were analyzed using the percolation theory (PT). The percolation threshold can be predicted, but it may strongly depend on the shape and the size of the nanofillers (32-34).

Generally, spherical shaped nanoparticles lead to higher values of critical concentration when compared to fillers with a high aspect-ratio (35). However, in this case the nanostructured arrangement of the nanofillers (i.e. the chain-like agglomerates) in the matrix could play a crucial role in the value of the percolation threshold. The random establishment of conductive nanostructures due to the nanofillers arrangement generated a non-detectable local electric field, rendering the prediction of the dielectric response of a nanocomposite complex $(36,9)$.

Our experimental data were also fitted using two different effective media approximation models (EMA). Their use implies a uniform dispersion of the nanofillers into the polymeric host with no local field fluctuation and neglecting the particles long-range electrostatic interaction (37-40). Such approximations were thus not suitable for the (Ni@C)PyrPS/SU-8 nanocomposites as it is observed on figure 8.a where Maxwell-Garnet (MG) (41) and Bruggeman (BS) (42) curves did not follow the trend in the permittivity increase with the filler volume fraction.

As a matter of fact, such approximations involve substantial variations in the prediction of the dielectric constant of a nanocomposite. Nevertheless, according to several studies (43-45), it is possible to estimate the value of the percolation threshold and thus predict the highest value of complex dielectric permittivity and complex conductivity by using a simple power laws as follow:

$$
\varepsilon^{*}=\varepsilon_{0}\left(\frac{\varphi_{c}-\varphi}{\varphi_{c}}\right)^{-s} \quad \text { for } \varphi<\varphi_{c}
$$




$$
\sigma^{*}=\sigma_{0}\left(\frac{\varphi_{c}-\varphi}{\varphi_{c}}\right)^{-t}
$$

Where $\varepsilon^{*}$ is the effective complex dielectric permittivity of the nanocomposite, $\varepsilon_{0}$ thedielectric permittivity of the polymeric host, $\varphi$ the filling fraction, $\varphi_{c}$ is the critical value of the percolation threshold and $s>0$ a parameter which takes into account the fractal size of the particles and their packing order. As consequence $\sigma^{*}$ is the effective complex AC conductivity of the nanocomposite, $\sigma_{0}$ the AC conductivity of the polymeric host and $t<2$ is the critical exponent. As stated by the previously mentioned works, critical values of the percolation threshold can vary within $0.3<s<1.8$ and $0.6<t<2$ respectively according to the specific conductive microstructures.

Figure 8 shows the compositional dependence of the dielectric parameters of (Ni@C)PyrPS/SU-8 nanocomposites obtained at $5 \mathrm{kHz}$.

(a)

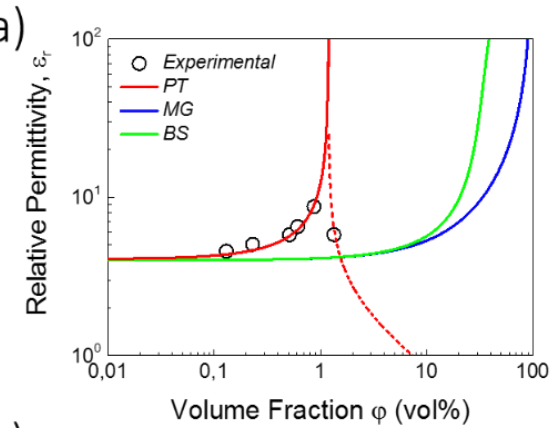

(c)

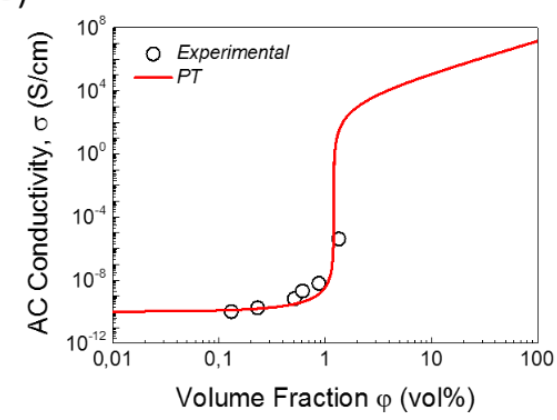

(b)

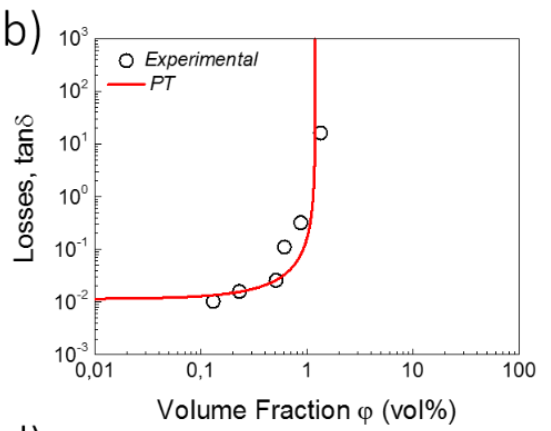

(d)

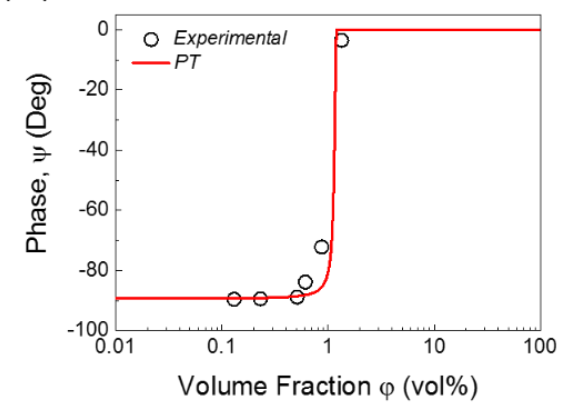

Figure 8 - Compositional dependence of the complex dielectric permittivity. Experimental values obtained at $5 \mathrm{kHz}$ : (a) permittivity, (b) loss tangent, (c) AC conductivity, (d) phase angle of (Ni@C)PyrPS/SU-8 nanocomposites. 
As shown in Figure 8.a, equations 3 and 4 fits the experimental data providing a percolation threshold value of $\varphi_{c}=1.2$ with the critical exponent $s=0.6$ and $t=1.8$ in agreement with the literature $(46,47)$.

The obtained percolation threshold (at 1.2 vol\%) was much lower than the theoretically predicted threshold (16-20 vol\%) according the EMA models, nevertheless it was in agreement with the percolation theory for high aspect-ratio nanofillers (48-50). This result can be easily attributed to the high aspect ratios of the chain-like agglomerates of (Ni@C) nanoparticles. Moreover, we highlight the fact that the value of percolation threshold obtained from 1.2 vol\% through equation 3 and 4 is in agreement with the experimental data.

\subsection{High-voltage characterization}

The results of high-voltage dielectric characterization of the devices are shown in Figure 9. Permittivity and loss tangent were measured with a bias up to $380 V_{D C}$ with a modulation of $20 V_{A C}$ at a fixed frequency of $5 \mathrm{kHz}$ (the sum of $A C$ and $D C$ voltage is limited to $\pm 400 \mathrm{~V}$ ). Note that the maximum electric field may vary between 0.27 and $0.38 \mathrm{MV} / \mathrm{cm}$ depending on the film thickness in the different devices.

In detail, Figure 9 shows superimposed data set with maximum DC bias voltages of $100 \mathrm{~V}, 200 \mathrm{~V}$, $300 \mathrm{~V}$ and $380 \mathrm{~V}$, respectively. No significant impact of the bias voltage used up to $380 \mathrm{~V}$ was visible on the permittivity and the loss tangent below a volume fraction of 0.51 vol\%.

In contrast, at 0.51 vol\%, the dependence of the permittivity with the electric field was not perfectly flat at the ends, and a "butterfly"-like loop could be clearly observed on the loss tangent. This abrupt change was an indication of a rising of additional dissipation loss that is fielddependent when the volume fraction became large.

This dissipation phenomenon had a hysteresis and remained reversible up to $300 \mathrm{~V} D$. At $380 \mathrm{~V}_{\mathrm{DC}}$, the capacitors were permanently damaged, evidence that could be assimilated with the dielectric 
breakdown. The high-voltage dielectric characterization was completed with current density measurements. Consistently with the abrupt change of loss tangent at 0.51 vol\% nanoparticle/polymer, we observed a drastic change of the field-dependent current density with a 4 orders of magnitude increase. The non-linear dependence of the leakage current density could be expressed as the Napierian logarithm as a function of the inverse of the applied electric field according to works (51-54).

(a)

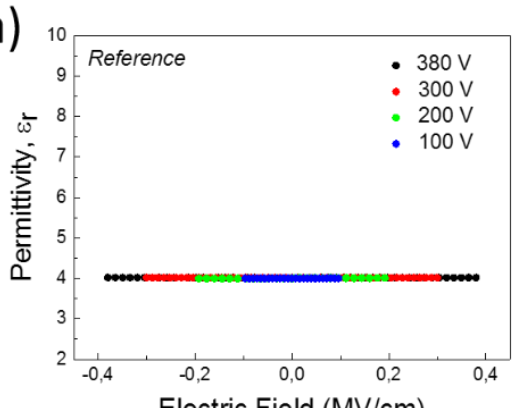

(c)

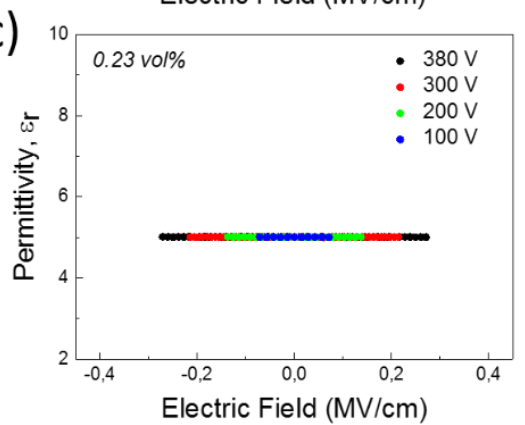

(e)

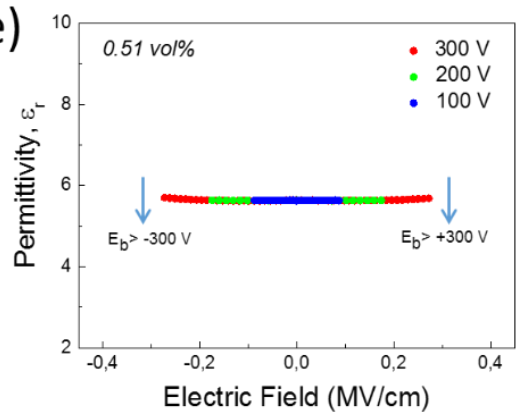

(b)

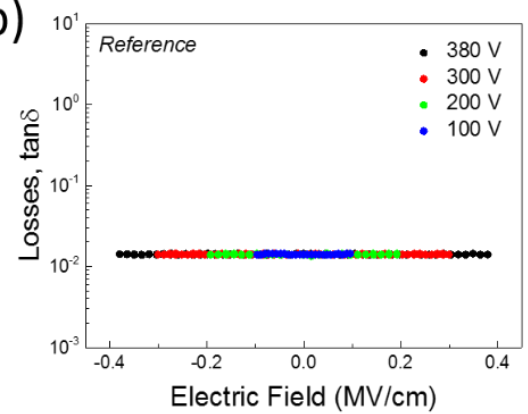

(d)

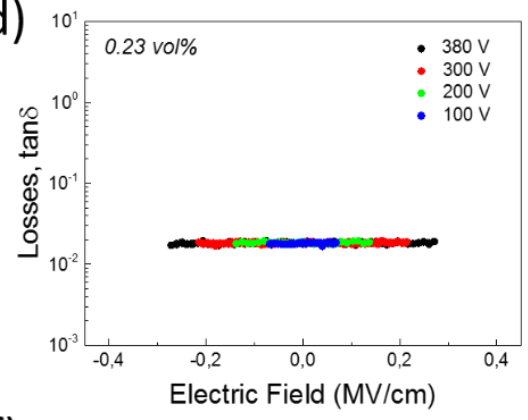

(f)

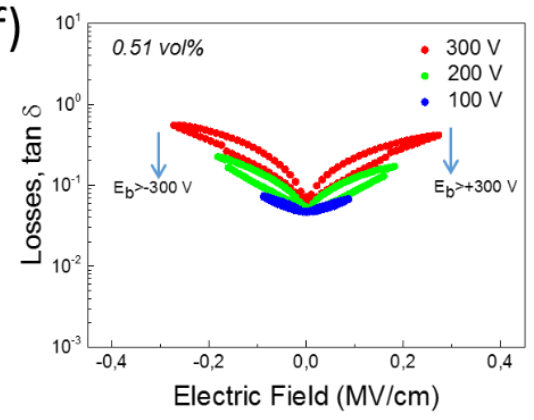

Figure 9 - High-voltage dielectric characterization. Permittivity and loss tangent vs DC bias field (maximum DC voltage $\pm 380 \mathrm{~V}$ ) at $5 \mathrm{kHz}$ : $(\mathrm{a}, \mathrm{b})$ pure SU-8 with a film thickness of $10.18 \mu \mathrm{m},(\mathrm{c}, \mathrm{d}$ ) (Ni@C)PyrPS/SU-8 (0.23 vol\%) with a film thickness of $15.54 \mu \mathrm{m},(\mathrm{e}, \mathrm{f})$ (Ni@C)PyrPS/SU-8 (0.51 vol\%) with a film thickness of $10.38 \mu \mathrm{m}$. 
This allowed to qualitatively separate two conduction mechanisms at 0.51 vol\% with a critical field ( $E_{\text {Trans }}$ ) of the order of $0.11 \mathrm{MV} / \mathrm{cm}$. The first mechanism was the direct electron tunneling (DT) and the second mechanism taking over at higher voltage was the Fowler-Nordheim tunneling (FNT). We may therefore assume that the enlarged dissipation loss at 0.51 vol\% were mainly caused by direct electron tunneling between nanoparticles.

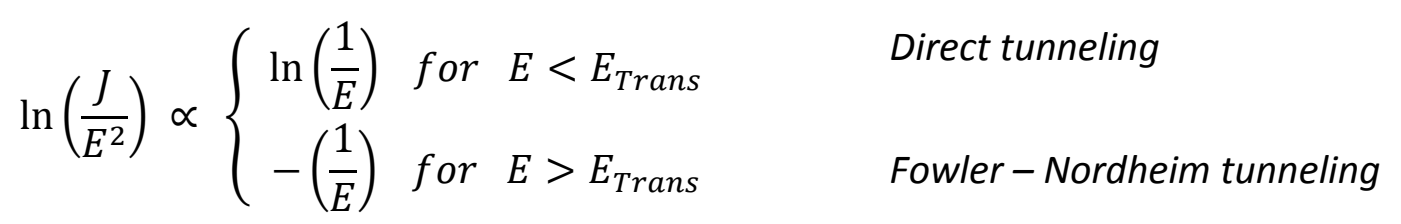

where $E_{\text {Trans }}$ could be derived from the position of the inflection point as shown in Figure 10.b.

It is interesting to note that this inflection point was not present in the formulated nanocomposites with lower filling fractions. This could be attributed to the greater average distance between the nanoparticles, which prevented this tunneling mechanism even at high electric field. Further focused ion beam SEM (FIB-SEM) tomography studies, will be needed to evaluate these mean distances between nanoparticles to confirm the above. Finally, even with this sudden increase in conduction, at large nanoparticles/polymer volume fraction there was no evidence of electrical aging or dielectric breakdown up to $\pm 0.38 \mathrm{MV} / \mathrm{cm}$. High-voltage results properties are summarized in Table 3.

(a)

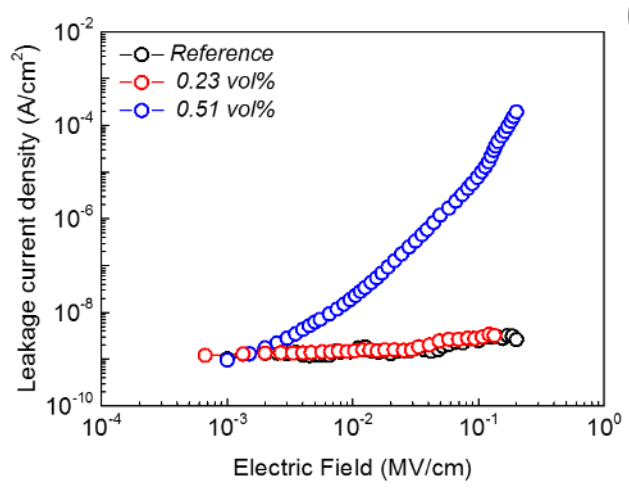

(b)

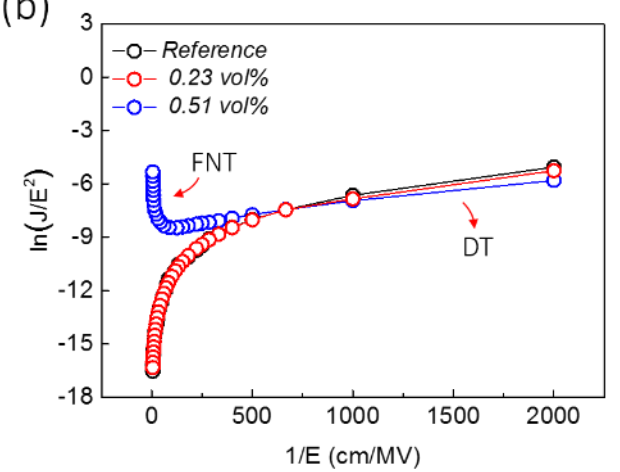

Figure 10 - Conduction mechanisms of the formulated nanocomposites - (a) Leakage current density as a function of the applied electric field for pure SU-8 $(10 \mu \mathrm{m})$, (Ni@C)PyrPS/SU-8 dielectric nanocomposite film of 0.23 vol\% (thickness: $15 \mu \mathrm{m}$ ) and (Ni@C)PyrPS/SU-8 dielectric 
nanocomposite film of 0.51 vol\% (thickness: $10 \mu \mathrm{m}$ ). (b) Fowler-Nordheim field emission mechanism and direct tunneling mechanism for the formulated nanocomposites as a function of the applied electric field.

Table 3 - High voltage dielectric properties at $5 \mathrm{KHz}$ with DC bias voltage

\begin{tabular}{|c|c|c|c|c|c|c|c|c|}
\hline \multirow[b]{2}{*}{$20 V_{A C}+V_{D C}$} & \multicolumn{4}{|c|}{ Permittivity, $\varepsilon_{r}$} & \multicolumn{4}{|c|}{ Energy Losses, $\tan \delta$} \\
\hline & $100 v_{D C}$ & $200 V_{D C}$ & $300 V_{D C}$ & $380 V_{D C}$ & $100 v_{D C}$ & $200 V_{D C}$ & $300 V_{D C}$ & $380 V_{D C}$ \\
\hline Reference & 4.04 & 4.04 & 4.04 & 4.04 & $1.11 \times 10^{-3}$ & $1.11 \times 10^{-3}$ & $1.11 \times 10^{-3}$ & $1.11 \times 10^{-3}$ \\
\hline $0.23 \mathrm{vol} \%$ & 5.10 & 5.10 & 5.10 & 5.10 & $1.80 \times 10^{-2}$ & $1.80 \times 10^{-2}$ & $1.80 \times 10^{-2}$ & $1.80 \times 10^{-2}$ \\
\hline \multirow{2}{*}{0.51 vol\% } & & & & & $4.0 \times 10^{-2}(\mathrm{~min})$ & $5.8 \times 10^{-2}(\mathrm{~min})$ & $7.9 \times 10^{-2}(\mathrm{~min})$ & Dielectric \\
\hline & 5.92 & 5.92 & 5.92 & 5.92 & $7.5 \times 10^{-2}(\max )$ & $2.1 \times 10^{-1}(\max )$ & $5.9 \times 10^{-1}(\max )$ & Breakdown \\
\hline
\end{tabular}

\section{Conclusion}

In this study we proposed a reliable wafer-scale film fabrication process for plate capacitors on silicon using sonodispersion and spin-coating techniques. The obtained results through dielectric spectroscopy were modeled using the generalized power-law equation of the percolation theory. The theoretical percolation threshold $\left(\varphi_{c}\right)$ was determinated to be close to 1.2 vol\%.

Three dielectric regimes were distinguished. In the first regime $\left(<\frac{1}{3} \varphi_{c}\right)$, the dielectric properties remained close to those of SU-8 with an increase in permittivity of about $25 \%$. In the second regime (between $\frac{1}{3} \varphi_{c}$ and $\frac{2}{3} \varphi_{c}$ ), the increase of permittivity was more significant, reaching $81 \%$ at a nanoparticles/polymer volume fraction of 0.61 vol\%. Moreover, the loss tangent values did not exceed $10^{-2}$. In the third regime (between $\frac{2}{3} \varphi_{c}$ and $\varphi_{c}$ ), the conductivity suddenly increased and the capacitor phase shifted towards zero.

However, no giant permittivity was reported in the vicinity of the percolation threshold although an enhancement of $116 \%$ was detected. High-voltage dielectric properties were also reported for the second regime revealing two electron field emission mechanisms. First, the direct tunneling (DT) equation correctly predicted an increase current density due to field emission below 0.11 
$\mathrm{MV} / \mathrm{cm}$. Second, Fowler-Nordheim (FN) model prevailed above $0.11 \mathrm{MV} / \mathrm{cm}$ in agreement with a steeper increase of the current density. This translated to the loss tangent with a "butterfly" pattern. No significant electrical aging or dielectric breakdown have occurred below $\pm 0.38 \mathrm{MV} / \mathrm{cm}$. Finally, SEM observations revealing typical chain-like agglomerates being perpendicular to the electrodes support the assumption that they might be held responsible of the low percolation threshold with (Ni@C). This strongly hindered the dielectric improvement of the MPC using (Ni@C). Furthermore, the role of the surface functionalization of $(\mathrm{Ni@C})$ with PyrPS as an electrical insulation barrier between particles remains questionable with regard to the exact nature of the carbon coating with (Ni@C).

In addition, as observed in the TEM images, the graphene-like coating shell does not appear on the smaller particles. However, these results provide a new path to improve the dielectric properties of nanocomposite photoresists suitable for the fabrication of miniaturized capacitors.

\section{References}

[1] F. Li, Q.-M. Wang, Array of dielectric nanocomposite devices using photoepoxy Su-8 as the polymeric phase, Appl. Phys. Lett. 89 (2006) 232905. https://doi.org/10.1063/1.2402885.

[2] A. Toor, H. So, A.P. Pisano, Dielectric properties of ligand-modified gold nanoparticle/SU-8 photopolymer based nanocomposites, Appl. Surf. Sci. 414 (2017) 373-379. https://doi.org/10.1016/i.apsusc.2017.04.096.

[3] J. Xu, C.P. Wong, High dielectric constant SU8 composite photoresist for embedded capacitors, J. Appl. Polym. Sci. 103 (2007) 1523-1528. https://doi.org/10.1002/app.24957.

[4] Jianwen Xu, C.P. Wong, Photodefinable High-k SU8 Nanocomposite for Embedded Capacitors, in: 2006 11th Int. Symp. Adv. Packag. Mater. Process. Prop. Interface, IEEE, Atlanta, GA, USA, 2006: pp. 133-138. https://doi.org/10.1109/ISAPM.2006.1666022.

[5] R.R. Navan, K. Prashanthi, M. Shojaei Baghini, V. Ramgopal Rao, Solution processed photopatternable high-k nanocomposite gate dielectric for low voltage organic field effect transistors, Microelectron. Eng. 96 (2012) 92-95. https://doi.org/10.1016/i.mee.2012.02.045.

[6] C. Mendes-Felipe, J.C. Barbosa, S. Gonçalves, N. Pereira, C.M. Costa, J.L. Vilas-Vilela, S. Lanceros-Mendez, High dielectric constant UV curable polyurethane acrylate/indium tin oxide composites for capacitive sensing, Compos. Sci. Technol. 199 (2020) 108363. https://doi.org/10.1016/i.compscitech.2020.108363.

[7] J. Chen, J. Liu, L. Cai, C. Wang, L. Liu, Q. Yang, C. Xiong, Incorporation of elaborately Synthesized BNNSs by a mild mechanical stirring process for the concurrent enhancement of thermal conductivity and dielectric breakdown strength of PVDF, Compos. Sci. Technol. 200 (2020) 108381. https://doi.org/10.1016/i.compscitech.2020.108381. 
[8] S.A. Zavyalov, A.A. Timofeev, A.N. Pivkina, J. Schoonman, Metal-polymer Nanocomposites: Formation and Properties Near the Percolation Threshold, in: P. Knauth, J. Schoonman (Eds.), Nanostructured Mater., Kluwer Academic Publishers, Boston, 2004: pp. 97-113. https://doi.org/10.1007/0-306-47722-X 6.

[9] L. Zhang, P. Bass, Z.-Y. Cheng, Revisiting the percolation phenomena in dielectric composites with conducting fillers, Appl. Phys. Lett. $105 \quad$ (2014) 042905. https://doi.org/10.1063/1.4892000.

[10] Z.-M. Dang, Y.-H. Lin, C.-W. Nan, Novel Ferroelectric Polymer Composites with High Dielectric Constants, Adv. Mater. 15 (2003) 1625-1629. https://doi.org/10.1002/adma.200304911.

[11] L. Zhang, W. Wang, X. Wang, P. Bass, Z.-Y. Cheng, Metal-polymer nanocomposites with high percolation threshold and high dielectric constant, Appl. Phys. Lett. 103 (2013) 232903. https://doi.org/10.1063/1.4838237.

[12] G.R. Achary P, M. B, S. Deo S, P. Ks, Study of Electrical Properties of Nickel Doped Polyurethane Nanocomposites, Polym. Sci. 04 (2018). https://doi.org/10.4172/2471-9935.100035.

[13] H. Takacs, B. Viala, J.-H. Tortai, V. Hermán, F. Duclairoir, Non -conductive ferromagnetic carboncoated (Co, Ni) metal/polystyrene nanocomposites films, J. Appl. Phys. 119 (2016) 093907. https://doi.org/10.1063/1.4942862.

[14] A. Plyushch, J. Macutkevič, J. Banys, P. Kuzhir, N. Kalanda, A. Petrov, C. Silvestre, M. Uimin, A. Yermakov, O. Shenderova, Carbon-Coated Nickel Nanoparticles: Effect on the Magnetic and Electric Properties of Composite Materials, Coatings. 8 (2018) 165. https://doi.org/10.3390/coatings8050165.

[15] X. Chen, F. Liang, W. Lu, Z. Jin, Y. Zhao, M. Fu, High Permittivity Nanocomposites Embedded with $\mathrm{Ag} / \mathrm{TiO} 2$ Core-Shell Nanoparticles Modified by Phosphonic Acid, Polymers. 10 (2018) 586. https://doi.org/10.3390/polym10060586.

[16] G. Chen, X. Wang, J. Lin, W. Yang, H. Li, Y. Wen, L. Li, Z. Jiang, Q. Lei, Nano-KTN@Ag/PVDF composite films with high permittivity and low dielectric loss by introduction of designed KTN/Ag core/shell nanoparticles, J. Mater. Chem. C. 4 (2016) 8070-8076. https://doi.org/10.1039/C6TC02321E.

[17] R. Fuhrer, E.K. Athanassiou, N.A. Luechinger, W.J. Stark, Crosslinking Metal Nanoparticles into the Polymer Backbone of Hydrogels Enables Preparation of Soft, Magnetic Field-Driven Actuators with MuscleLike Flexibility, Small. 5 (2009) 383-388. https://doi.org/10.1002/smll.200801091.

[18] H. Takacs, B. Viala, V. Herman, J. Alarcon Ramos, J.-H. Tortai, F. Duclairoir, New Approach to Closely Spaced Disordered Cobalt-Graphene Polymer Nanocomposites for Non-Conductive RF Ferromagnetic Films, IEEE Trans. Magn. $51 \quad$ (2015) 1-4. https://doi.org/10.1109/TMAG.2015.2445824.

[19] F. He, S. Lau, H.L. Chan, J. Fan, High Dielectric Permittivity and Low Percolation Threshold in Nanocomposites Based on Poly(vinylidene fluoride) and Exfoliated Graphite Nanoplates, Adv. Mater. 21 (2009) 710-715. https://doi.org/10.1002/adma.200801758.

[20] Z. Zeng, C. Wang, Y. Zhang, P. Wang, S.I. Seyed Shahabadi, Y. Pei, M. Chen, X. Lu, Ultralight and Highly Elastic Graphene/Lignin-Derived Carbon Nanocomposite Aerogels with Ultrahigh Electromagnetic Interference Shielding Performance, ACS Appl. Mater. Interfaces. 10 (2018) 82058213. https://doi.org/10.1021/acsami.7b19427.

[21] R. Hashemi, G.J. Weng, A theoretical treatment of graphene nanocomposites with percolation threshold, tunneling-assisted conductivity and microcapacitor effect in $A C$ and DC electrical settings, Carbon. 96 (2016) 474-490. https://doi.org/10.1016/j.carbon.2015.09.103.

[22] Y. Jiao, L. Yuan, G. Liang, A. Gu, Facile Preparation and Origin of High- k Carbon Nanotube/Poly(Ether Imide)/Bismaleimide Composites through Controlling the Location and Distribution of Carbon Nanotubes, J. Phys. Chem. C. 118 (2014) 24091-24101. https://doi.org/10.1021/ip506482s. 
[23] X. Xia, Z. Zhong, G.J. Weng, Maxwell-Wagner-Sillars mechanism in the frequency dependence of electrical conductivity and dielectric permittivity of graphene-polymer nanocomposites, Mech. $\quad$ Mater. $109 \quad$ (2017) 42-50. https://doi.org/10.1016/i.mechmat.2017.03.014.

[24] Z.-H. Dai, T. Li, Y. Gao, J. Xu, J. He, Y. Weng, B.-H. Guo, Achieving high dielectric permittivity, high breakdown strength and high efficiency by cross-linking of poly(vinylidene fluoride)/BaTiO3 nanocomposites, Compos. Sci. Technol. 169 (2019) 142-150. https://doi.org/10.1016/i.compscitech.2018.10.014.

[25] J. Zhang, F. Zhao, Y. Zuo, Y. Zhang, X. Chen, B. Li, N. Zhang, G. Niu, W. Ren, Z. Ye, Improving actuation strain and breakdown strength of dielectric elastomers using core-shell structured CNT-Al2O3, Compos. Sci. Technol. $200 \quad$ (2020) 108393. https://doi.org/10.1016/i.compscitech.2020.108393.

[26] Z.-M. Dang, J.-K. Yuan, S.-H. Yao, R.-J. Liao, Flexible Nanodielectric Materials with High Permittivity for Power Energy Storage, Adv. Mater. 25 (2013) 6334-6365. https://doi.org/10.1002/adma.201301752.

[27] D. Wang, X. Zhang, J.-W. Zha, J. Zhao, Z.-M. Dang, G.-H. Hu, Dielectric properties of reduced graphene oxide/polypropylene composites with ultralow percolation threshold, Polymer. 54 (2013) 1916-1922. https://doi.org/10.1016/i.polymer.2013.02.012.

[28] J. Liu, G. Tian, S. Qi, Z. Wu, D. Wu, Enhanced dielectric permittivity of a flexible three-phase polyimide-graphene-BaTiO3 composite material, Mater. Lett. 124 (2014) 117-119. https://doi.org/10.1016/i.matlet.2014.02.105.

[29] L.L. Vovchenko, O.V. Lozitsky, V.V. Oliynyk, V.V. Zagorodnii, T.A. Len, L.Y. Matzui, Yu.S. Milovanov, Dielectric and microwave shielding properties of three-phase composites graphite nanoplatelets/carbonyl iron/epoxy resin, Appl. Nanosci. 10 (2020) 4781-4790. https://doi.org/10.1007/s13204-020-01326-w.

[30] P. Fan, L. Wang, J. Yang, F. Chen, M. Zhong, Graphene/poly(vinylidene fluoride) composites with high dielectric constant and low percolation threshold, Nanotechnology. 23 (2012) 365702. https://doi.org/10.1088/0957-4484/23/36/365702.

[31] L. Cui, X. Lu, D. Chao, H. Liu, Y. Li, C. Wang, Graphene-based composite materials with high dielectric permittivity via an in situ reduction method: Graphene-based composite materials with high dielectric permittivity, Phys. Status Solidi A. 208 (2011) 459-461. https://doi.org/10.1002/pssa.201026273.

[32] I. Balberg, N. Binenbaum, Computer study of the percolation threshold in a twodimensional anisotropic system of conducting sticks, Phys. Rev. B. 28 (1983) 3799-3812. https://doi.org/10.1103/PhysRevB.28.3799.

[33] A. Celzard, E. McRae, C. Deleuze, M. Dufort, G. Furdin, J.F. Marêché, Critical concentration in percolating systems containing a high-aspect-ratio filler, Phys. Rev. B. 53 (1996) 6209-6214. https://doi.org/10.1103/PhysRevB.53.6209.

[34] S.I. Heo, J.C. Yun, K.S. Oh, K.S. Han, Influence of particle size and shape on electrical and mechanical properties of graphite reinforced conductive polymer composites for the bipolar plate of PEM fuel cells, Adv. Compos. Mater. $15 \quad$ (2006) 115-126. https://doi.org/10.1163/156855106776829356.

[35] Q. Xue, The influence of particle shape and size on electric conductivity of metal-polymer composites, Eur. Polym. J. 40 (2004) 323-327. https://doi.org/10.1016/j.eurpolymj.2003.10.011.

[36] L. Zhang, Z.-Y. Cheng, DEVELOPMENT OF POLYMER-BASED 0-3 COMPOSITES WITH HIGH DIELECTRIC CONSTANT, J. Adv. Dielectr. 01 (2011) 389-406. https://doi.org/10.1142/S2010135X11000574. 
[37] S. Giordano, Effective medium theory for dispersions of dielectric ellipsoids, J. Electrost. 58 (2003) 59-76. https://doi.org/10.1016/S0304-3886(02)00199-7.

[38] M. Siddiqui, A. Arif, Generalized Effective Medium Theory for Particulate Nanocomposite Materials, Materials. 9 (2016) 694. https://doi.org/10.3390/ma9080694.

[39] S. Li, R. Chen, S. Anwar, W. Lu, Y. Lai, H. Chen, B. Hou, F. Ren, B. Gu, Applying effective medium theory in characterizing dielectric constant of solids, Prog. Electromagn. Res. Lett. 35 (2012) 145-153. https://doi.org/10.2528/PIERL12072108.

[40] R.E. Diaz, W.M. Merrill, N.G. Alexopoulos, Analytic framework for the modeling of effective media, J. Appl. Phys. 84 (1998) 6815-6826. https://doi.org/10.1063/1.369013.

[41] V.A. Markel, Introduction to the Maxwell Garnett approximation: tutorial, J. Opt. Soc. Am. A. 33 (2016) 1244. https://doi.org/10.1364/JOSAA.33.001244.

[42] D.A.G. Bruggeman, Berechnung verschiedener physikalischer Konstanten von heterogenen Substanzen. II. Dielektrizitätskonstanten und Leitfähigkeiten von Vielkristallen der nichtregulären Systeme, Ann. Phys. 417 (1936) 645-672. https://doi.org/10.1002/andp.19364170706.

[43] J. Yacubowicz, M. Narkis, Dielectric behavior of carbon black filled polymer composites, Polym. Eng. Sci. 26 (1986) 1568-1573. https://doi.org/10.1002/pen.760262207.

[44] M.T. Clarkson, Electrical conductivity and permittivity measurements near the percolation transition in a microemulsion. II. Interpretation, Phys. Rev. A. 37 (1988) 2079-2090. https://doi.org/10.1103/PhysRevA.37.2079.

[45] C.-W. Nan, PHYSICS OF INHOMOGENEOUS INORGANIC MATERIALS, Prog. Mater. Sci. 37 (1993) 1-116. https://doi.org/10.1016/0079-6425(93)90004-5.

[46] C. Pecharromun, F. Esteban-Betegón, J.F. Bartolom $\varnothing$, S. López-Esteban, J.S. Moya, New Percolative BaTiO3 $\pm \mathrm{Ni}$ Composites with a High and Frequency-Independent Dielectric Constant

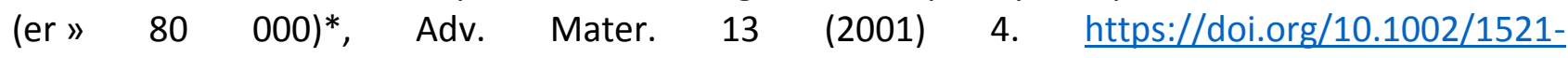
4095(200110)13:20<1541::AID-ADMA1541>3.0.CO;2-X.

[47] A. Ameli, M. Nofar, C.B. Park, P. Pötschke, G. Rizvi, Polypropylene/carbon nanotube nano/microcellular structures with high dielectric permittivity, low dielectric loss, and low percolation threshold, Carbon. 71 (2014) 206-217. https://doi.org/10.1016/j.carbon.2014.01.031. [58] S.C. Tjong, G.D. Liang, S.P. Bao, Electrical behavior of polypropylene/multiwalled carbon nanotube nanocomposites with low percolation threshold, Scr. Mater. 57 (2007) 461-464. https://doi.org/10.1016/i.scriptamat.2007.05.035.

[49] B.M. Mandal, Conducting polymer nanocomposites with extremely low percolation threshold, Bull. Mater. Sci. 21 (1998) 161-165. https://doi.org/10.1007/BF02927565.

[50] D. Nuzhnyy, M. Savinov, V. Bovtun, M. Kempa, J. Petzelt, B. Mayoral, T. McNally, Broadband conductivity and dielectric spectroscopy of composites of multiwalled carbon nanotubes and poly(ethylene terephthalate) around their low percolation threshold, Nanotechnology. 24 (2013) 055707. https://doi.org/10.1088/0957-4484/24/5/055707.

[51] M.H. Lean, W.-P.L. Chu, Modeling effect of nanofillers on charge transport in composite polymer films for energy storage, J. Energy Storage. 4 (2015) 36-50. https://doi.org/10.1016/j.est.2015.09.001.

[52] G. Zhang, Y. Li, S. Tang, R.D. Thompson, L. Zhu, The Role of Field Electron Emission in Polypropylene/Aluminum Nanodielectrics Under High Electric Fields, ACS Appl. Mater. Interfaces. 9 (2017) 10106-10119. https://doi.org/10.1021/acsami.7b00095.

[53] T. Ikuno, H. Okamoto, Y. Sugiyama, H. Nakano, F. Yamada, I. Kamiya, Electron transport properties of Si nanosheets: Transition from direct tunneling to Fowler-Nordheim tunneling, Appl. Phys. Lett. 99 (2011) 023107. https://doi.org/10.1063/1.3610486. 
[54] J. Melai, C. Salm, S. Smits, J. Visschers, J. Schmitz, The electrical conduction and dielectric strength of SU-8, J. Micromechanics Microengineering. $19 \quad$ (2009) 065012. https://doi.org/10.1088/0960-1317/19/6/065012. 